\title{
Aproximación teórica a las redes de cooperación en el marco de la actividad turística
}

\section{Theoretical approach to cooperation networks within the framework of the tourist activity}

Gutiérrez, Milagros

Narváez, Mercy

Universidad del Zulia, Venezuela

Autor para correspondencia: milagros_gutierrez@hotmail.com

Fecha de recepción: 01 de Junio de 2017 - Fecha de aceptación: 15 de Septiembre de 2017

Resumen: El actual dinamismo económico, tecnológico y cultural predominante a nivel mundial, ha llevado a los destinos turísticos a implementar estrategias para promover una oferta turística innovadora, competitiva y sustentable que les permita posicionarse en los mercados locales e internacionales. Una de estas estrategias es la integración pública - privada a través de redes de cooperación, las cuales permiten a los actores que hacen vida en los destinos, actuar sobre los factores de atracción, soporte y gestión, para fortalecer la posición competitiva de los mismos. En este marco referencial, el presente artículo tiene como propósito realizar una aproximación teórica a la conceptualización de las redes de cooperación, sus propiedades, objetivos e importancia; partiendo de la contrastación de enfoques teóricos vigentes. Para alcanzar este objetivo se utilizó una metodología de tipo descriptivo, basada en un enfoque constructivista, con un diseño de investigación documental. De la información analizada se concluye que las redes de cooperación constituyen mecanismos que facilitan a los actores turísticos el intercambio de recursos, información y servicios, con lo que se logran objetivos de desarrollo competitivo tales como el fortalecimiento del producto turístico, la planificación integral y sostenible del destino y la puesta en valor de los factores de atracción.

Palabras clave: redes; cooperación; sector turístico; competitividad

\begin{abstract}
The current global economic, technological and cultural dynamism has led tourist destinations to implement strategies to promote an innovative, competitive and sustainable tourism offer, that allows them to position themselves in local and international markets. One of these strategies is public - private integration through cooperation networks, which allow the actors who live in the destinations, act on the factors of attraction, support and management, to strengthen their competitive position. In this reference frame, this article aims to make a theoretical approach to the conceptualization of cooperation networks, their properties and objectives; starting from the contrast of current theoretical approaches. To reach this objective, a descriptive methodology was used, based on a constructivist approach, with a documental research design. From the analyzed information, it is concluded that the cooperation networks are mechanisms that facilitate the tourist actors the exchange of resources, information and services, which achieve competitive development objectives such as the strengthening of tourism product, integral and sustainable planning for the destination and the value of the attraction factors.
\end{abstract}

Key words: networks; cooperation; touristic destination; competitiveness 


\section{Introducción}

El turismo ha pasado a ser una actividad social y económica de gran importancia en los últimos años, por su potencial para contribuir con el desarrollo de los territorios; los cuales tratan de aprovechar sus recursos y potencialidades, generando a partir de los mismos, estrategias para promover una oferta turística competitiva y sustentable, que les permita posicionarse con éxito en los mercados nacionales e internacionales. Parte importante de este proceso recae en los actores privados que hacen vida en el territorio, por ser los encargados de ofrecer la gama de servicios y productos necesarios para satisfacer las necesidades de los visitantes; sin embargo, su actuación se encuentra conectada a la de otros grupos de actores, a la vez que es regulada por la gestión y el desarrollo de políticas por parte de los entes públicos con competencia en turismo.

Puede afirmarse entonces, que la integración y coordinación eficiente entre todos los grupos de actores resulta necesaria, si se pretenden alcanzar altos niveles de competitividad turística, traducidos en los máximos beneficios económicos y sociales para el enclave.

De allí que, una de las principales estrategias de integración pública - privada la constituyen las redes de cooperación, ya que, al promover el intercambio de información, recursos y servicios, necesarios para producir sinergias, permiten a los actores vinculados alcanzar objetivos individuales y colectivos, y a la vez promover el posicionamiento de los destinos turísticos, mediante el logro de objetivos que incluyen aspectos como la promoción del destino, el fortalecimiento del producto turístico, la captación de eventos para la localidad, la planificación sustentable e integral del destino y el incremento de la rentabilidad.

En este contexto referencial, el propósito de este artículo, es realizar una aproximación teórica a las redes de cooperación, en el marco de la actividad turística, desarrollando los principales elementos conceptuales referidos a esta temática: definiciones, propiedades y objetivos que pueden ser alcanzados a través de las mismas. Para ello se utilizó una metodología de tipo descriptiva y documental, basada en un enfoque constructivista, que abarcó tres fases, denominadas exploratoria, descriptiva y de construcción.

De la información analizada se concluye que, las redes de cooperación se conforman en los destinos turísticos como una estrategia de colaboración entre actores públicos y privados, con el propósito emprender acciones que permitan lograr objetivos orientados a posicionar el enclave turístico. Este tipo de estructura puede ser representada gráficamente a través de nodos (actores) e interconexiones (relaciones) y posee tres propiedades principales: intensidad relacional, centralidad y conectividad, las cuales al ser analizadas desde el punto de vista social, ofrecen información importante sobre las características del relacionamiento entre los actores.

\section{Metodología}

La investigación, desarrollada bajo un enfoque constructivista, se caracterizó por planteamientos resultantes de las construcciones intelectuales de las investigadoras, producto de su relación directa con el objeto de conocimiento (Cubero, 2005: 16). Se utilizó como técnica de recolección de datos la observación documental, consistente, según Trigueros y col. (2001: 124) en la observación y el análisis de textos escritos, documentos numéricos y estadísticos, y reproducciones de imagen y sonido. 
El proceso investigativo se desarrolló en tres fases:

La primera fase, denominada exploratoria, se inició con la sensibilización previa en torno a la importancia de las redes de cooperación organizacional, como estrategia para promover el desarrollo competitivo del sector turístico; lo cual colocó de manifiesto la necesidad de realizar una aproximación teórica a la conceptualización de las redes.

La segunda fase (descriptiva), comprendió el desarrollo de los principales aspectos teóricos asociados a las redes de cooperación y sus implicaciones en el desarrollo competitivo de los destinos turísticos, a partir de la indagación del conocimiento construido hasta el momento, representado en textos y artículos. Durante esta fase se empleó el método de análisis de contenido, y como técnica, la observación documental, la cual se realizó con base en la teoría fundamentada, como enfoque metodológico centrado en la premisa de que la teoría es indispensable para el conocimiento profundo de cualquier fenómeno social; siendo su objetivo, ayudar al investigador a generar proposiciones a través de procedimientos analíticos, para producir un conocimiento integrado, consistente, plausible y cercano a los datos (Kornblit, 2007).

La tercera fase, denominada "de construcción”, se llevó a cabo empleando los métodos de análisis de contenido, deducción e inducción, los cuales permitieron realizar inferencias de la información examinada, para construir argumentos propios acerca de las redes de cooperación y su importancia como estrategia para el desarrollo competitivo de los destinos turísticos.

\section{Argumentación Teórica}

\section{Conceptualización de las Redes de Cooperación Organizacional}

En el marco de las ciencias organizacionales, Vázquez (2005), define las redes empresariales como el sistema de relaciones y contactos, que vincula a empresas y actores entre sí, cuyo contenido puede referirse a bienes materiales, información o tecnología. Se trata de relaciones entre empresas o empresarios, que permiten el intercambio de bienes y servicios, o de aquellas informaciones que incorporan conocimientos.

Para Mifflin, citado por Bonomie y Meleán (2007), una red empresarial está dada por el conjunto de relaciones integradas, entre los agentes que participan en un aparato productivo; no refiriéndose el término, en particular, a asociaciones horizontales o verticales entre empresas, sino a la dinámica económica y social que se aprecia entre los diversos agentes, aglutinados en torno a una actividad productiva determinada. De esta manera, la creación de redes es producto de la cooperación o coalición de empresas, que buscan un objetivo común, donde cada participante mantiene independencia jurídica y gerencial.

Desde esta misma perspectiva, Becerra y Álvarez (2011) definen las redes empresariales como una forma de organizar las relaciones entre empresas, las cuales, sin dejar de competir, incluyen la cooperación como parte de su estrategia de negocio, con el propósito de alcanzar un mejor desempeño, en una determinada industria. 
Estos planteamientos son complementados por Fernández y Arranz (2010), al explicar que las redes consisten en una forma de cooperación que va más allá de las empresas, incorporando además a organizaciones. Por tratarse de un tipo de cooperación, se caracterizan por la existencia de una pluralidad de acuerdos entre los participantes, sean éstos empresas, o entre empresas y otro tipo de instituciones públicas y privadas. En este sentido, se destaca la importancia del papel que desempeñan las organizaciones públicas, como soportes críticos del desarrollo de las agrupaciones de empresas, para el fomento del crecimiento económico y social de una región.

Partiendo de las propuestas conceptuales anteriores, en las que se evidencia, en primer lugar, la posibilidad de conformar redes no sólo entre empresas, sino además entre empresas y organizaciones, o únicamente entre organizaciones con algún vínculo en común; y en segundo lugar, la importancia de la cooperación, como condición para la formación y el mantenimiento de las redes, se definen las redes de cooperación organizacional como:

Sistemas complejos y dinámicos, de relaciones e interacciones, establecidos entre actores sociales de carácter público y privado, para alcanzar objetivos comunes, a través de la cooperación y el intercambio de información, recursos, bienes y servicios.

En el marco de la actividad turística, las redes se forman por la asociación de organizaciones, tanto de carácter lucrativo como por organismos públicos e incluso por organizaciones no estatales, que cooperan entre sí con el propósito de promover, de manera conjunta y permanente los recursos económicos, sociales, culturales, naturales y humanos de una localidad, en aras de lograr un fin común de desarrollo turístico, para un destino determinado.

De los planteamientos anteriores, se derivan dos aspectos importantes, el primero de ellos referido a los actores sociales que conforman las redes, y el segundo tiene que ver con las relaciones de cooperación que se establecen entre estos actores.

En cuanto al primer aspecto, destaca Perego (2003) que al hablar de actores sociales, se hace referencia al Estado, las empresas, las organizaciones y los agentes económicos locales. Comenzando con el Estado, éste cumple un rol determinante para el funcionamiento de las redes, a través de su intervención en el ambiente de negocios, evidenciada en aspectos como el otorgamiento de licencias, la fijación y el cobro de impuestos y la administración de los servicios públicos, entre otros.

Las organizaciones y empresas, por su parte, dan forma al ambiente general de negocios, al atraer clientes, establecer relaciones con proveedores, contribuir con las mejoras de la infraestructura local, crear centros de capacitación y, en general, al vincularse con otras organizaciones, para articular acciones en pro del bienestar de la comunidad donde desarrollan sus actividades.

En relación con los agentes económicos locales, éstos se encuentran representados por las entidades públicas o privadas, cuya participación en la creación y el funcionamiento de las redes, se revela en aspectos como la promoción, el financiamiento, la formación de recursos humanos y 
la oferta de servicios especializados (empresariales y tecnológicos, entre otros), a fin de crear el clima y la infraestructura física necesaria, para apoyar el proceso de desarrollo de las redes organizacionales, con el consiguiente logro de objetivos. Forman parte de este grupo:

$\checkmark$ Organizaciones empresariales (cámaras empresariales, asociaciones de empresarios).

$\checkmark$ Entidades gubernamentales, a nivel local, regional o nacional.

$\checkmark$ Organismos financieros y bancas de desarrollo.

$\checkmark$ Instituciones del sector de educación superior.

$\checkmark$ Centros de servicios empresariales.

$\checkmark$ Organizaciones privadas no lucrativas.

$\checkmark$ Organismos internacionales.

Por último, las personas naturales, organizadas en comunidades, también forman parte importante de las redes de cooperación, sobre todo si las mismas son articuladas con el objetivo de desarrollar los destinos turísticos. Los habitantes de las localidades receptoras, a través sus valores, actitudes y apertura, tienen la capacidad de influir en la percepción del turista acerca del destino. Igualmente, la participación de los residentes en el proceso de planificación del destino, se torna necesaria en la medida que los resultados de este proceso los afecten o beneficien de alguna manera.

Así, considerando el rol que cada grupo de actores cumple en función de promover el desarrollo competitivo de los destinos, se infiere que la presencia de todos es necesaria en los procesos de articulación pública - privada que dan lugar a las redes.

La cooperación, por su parte, constituye un elemento común en todas las propuestas conceptuales mencionadas, de allí la necesidad de conceptualizarla y destacar sus principales características. Al respecto, Sáez y Cabanelas (1997) expresan que, cooperar consiste en compartir, voluntaria y recíprocamente algún recurso y/o conocimiento de tipo tecnológico, comercial o financiero, con el objeto de desarrollar una estrategia que redunde en ventajas competitivas para los cooperadores.

Consecuentemente, González (2007) plantea que el principal beneficio de la cooperación es el incremento de la competitividad de las empresas que se integran en procesos asociativos, a la vez que destaca las siguientes características de las redes de cooperación:

Selectividad: las organizaciones dentro de una red sólo cooperan con otros actores con los que logran determinar algún objetivo común.

Complementariedad: las empresas presentan mayor disposición a cooperar con otras empresas cuyas fortalezas y oportunidades permitan reducir las debilidades y amenazas propias.

Temporalidad: en líneas generales, las organizaciones están dispuestas a cooperar de forma transitoria, a corto plazo. No obstante, el período de cooperación puede prolongarse conforme se estrechan los vínculos entre las organizaciones y la confianza aumenta. En la medida que las empresas cooperadoras tengan éxito, renovarán los acuerdos y/o vínculos 
existentes y crearán nuevos, extendiendo cada vez más el plazo y el grado de compromiso de los mismos.

Contribución a la solución de problemas organizacionales: las redes de cooperación permiten a las organizaciones valerse de terceros, para llevar a cabo acciones que por sí solas no podrían realizar.

Se basan en la negociación y la confianza: la cooperación se negocia (no se logra a través de exigencias, ni es un acto solidario), porque con la misma se espera un incremento en la competitividad y rentabilidad de todas las partes involucradas; por tanto, su éxito implica tomar en cuenta las diferentes perspectivas y percepciones de todos los actores. La confianza, por su parte, se considera una variable no económica, que aunque difícilmente entra en el cálculo de las empresas, es estratégica para las relaciones económicas de mercado Vázquez (2005). Las transacciones económicas y comerciales entre actores, con o sin contratos formales, se basan en la expectativa mutua de las partes, de que el otro es confiable y cumplirá con lo acordado. Así, si se quieren aprovechar al máximo los vínculos de cooperación, la confianza debe ser fomentada creando un clima correcto, vigilando las aportaciones de los actores cooperadores y cuidando las relaciones internas (Sáez y Cabanelas, 1997).

\section{Componentes, Topología y Propiedades de las Redes de Cooperación.}

Desde el punto de vista morfológico, las redes pueden ser representadas a través de grafos conformados por nodos e interconexiones. Los nodos simbolizan a los diferentes actores que forman parte de la red; en tanto que las interconexiones representan las relaciones que se producen entre los actores. La disposición de estos dos componentes puede dar lugar a tres tipos de estructuras reticulares:

Estrella: Bajo esta configuración, diferentes organizaciones establecen vínculos de cooperación simples simultáneos, con una organización central que asume el liderazgo. Cuanto más pequeña sea una red y más débiles los vínculos de cooperación, mayor será su tendencia a tomar la forma de estrella (González, 2007).

Mixta: Este tipo de red, es conformada por organizaciones que establecen entre sí vínculos simples y múltiples de manera simultánea. En la medida que los vínculos entre los actores se debiliten, la red mixta tenderá a tomar forma de estrella; mientras que, la red con forma de estrella tenderá a tomar la forma mixta, cuando los vínculos entre los actores se consoliden, como consecuencia de la confianza mutua y los buenos resultados que obtengan las organizaciones al cooperar.

Malla: Se caracteriza por la rotación del liderazgo entre los distintos proyectos. Los roles de los actores son intercambiables, la jerarquía es nula, la flexibilidad es máxima y el riesgo de interrupción de los vínculos es mínimo. Aclara González ob.cit. (2007) que, este tipo de red es una estructura ideal, representativa de un mercado donde no existen vínculos competitivos y los actores mantienen relaciones de cooperación estrecha, permanente, bajo un clima de confianza absoluta. 


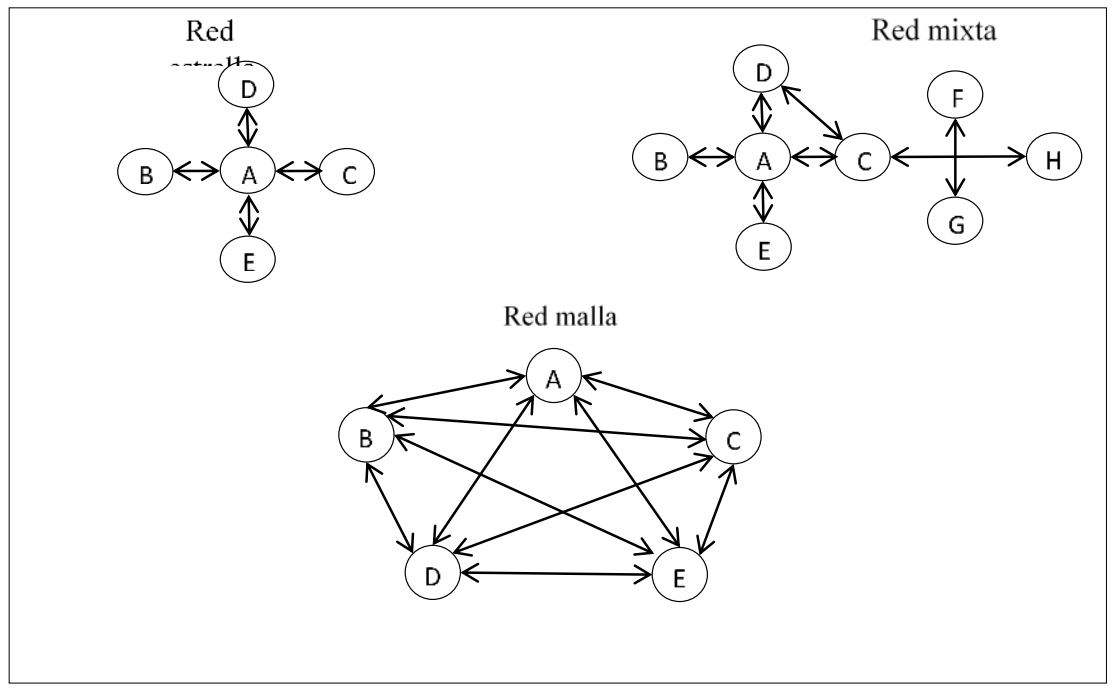

Figura 1. Topología de las redes de cooperación.

Ahora bien, el análisis de las estructuras presentadas, lleva a distinguir en las redes tres propiedades:

Intensidad relacional: proporciona información acerca del número total de conexiones establecidas entre los actores de la red. Cuanto mayor es la cantidad de conexiones de un actor, mayor es su capacidad de acción dentro de la red.

Centralidad: un actor es central en la medida que se encuentra implicado en todas las conexiones de la red o asume un rol de mediación entre los actores. Los actores que ocupan posiciones centrales tienen mayor capacidad para incidir en la opinión y conducta de otros actores (Garrido, 1988), y pueden además controlar la generación de contactos y el flujo de información.

Conectividad: las relaciones entre los actores de la red se fundamentan en la existencia de vínculos, cuya direccionalidad y fuerza otorgan a las mismas cualidades específicas. Con respecto a la direccionalidad, ésta se refiere a las conexiones recíprocas o en un solo sentido que pueden tener lugar entre los actores. La fuerza, por su parte, es determinada por la cantidad y multiplicidad de contenido transferido entre las conexiones (Granovetter, 1973). Se considera además, como medida válida para medir la fortaleza de los vínculos, la cantidad de contactos establecidos por los actores en períodos de tiempo determinados.

\section{Clasificación de las Redes de Cooperación.}

Galán y col. (2010) proponen la clasificación de las redes de cooperación a partir del análisis de investigaciones previas realizadas sobre el tema. Así, destacan que Hinterhuber y Levin (1994) clasifican las redes en cuatro tipos: redes internas de las organizaciones, redes verticales conformadas por organizaciones que se desempeñan a lo largo de un proceso productivo (hacia arriba y hacia abajo); redes horizontales, basadas en alianzas que se establecen entre organizaciones similares en mercados parecidos, y redes diagonales, formadas entre 
organizaciones de distintos sectores, que tratan de explotar sinergias para crear nuevos e interdisciplinares mercados.

Por otro lado, Grandori y Soda (1995) proponen que según el grado de formalización en los acuerdos e intercambios entre las organizaciones que conforman las redes, éstas pueden ser sociales, cuando las relaciones entre los actores son personales e informales; burocráticas, cuando las relaciones no son sólo personales e informales, sino que también son institucionales; y de propiedad, cuando tienen una unidad central de coordinación.

Astley y Fombrun (1983), aplicando el modelo biológico al campo de las organizaciones, explican que según la forma de interdependencia entre las organizaciones, existen redes de relaciones comensalistas, producidas entre empresas de un mismo sector y redes de relaciones simbióticas, que se generan entre organizaciones de diferentes sectores. Por otra parte, estos autores plantean otra clasificación, según el tipo de asociación entre los actores, que puede ser directa (cuando los actores se relacionan directamente) o indirecta (cuando los actores se relacionan a través de otros actores que hacen de intermediarios). La integración entre ambas dimensiones (interdependencia y tipo de asociación) da lugar a cuatro tipos de redes posibles entre organizaciones, a saber:

Redes aglomeradas: están conformadas por organizaciones de un mismo sector, con relaciones débiles o indirectas, que realizan movimientos conjuntos para intercambiar información y para obtener un posicionamiento común en los mercados.

Redes confederadas: se componen por organizaciones de un mismo sector, que establecen relaciones fuertes y directas. En estas relaciones se combina la cooperación con la competencia y existen acuerdos formales e informales que sirven para dirigir las acciones conjuntas.

Redes conjugadas: están formadas por empresas de sectores distintos, con vínculos directos y fuertes. Normalmente cuentan con vínculos jerárquicos.

Redes orgánicas: se dan entre empresas de sectores distintos con relaciones indirectas. Los miembros de este tipo de red realizan acciones conjuntas, apoyados en organizaciones externas, generalmente públicas.

\begin{tabular}{|c|c|c|}
\hline Tipos de asociación & \multicolumn{2}{|c|}{ Formas de interdependencia } \\
\cline { 2 - 3 } & Comensalista & Simbiótica \\
\hline Directa & Confederada & Conjugada \\
\hline Indirecta & Aglomerada & Orgánica \\
\hline
\end{tabular}

Cuadro 1. Tipología de las Redes de Organizaciones, según Astley y Fombrun (1983)

Las Redes de Cooperación como Estrategia para el Desarrollo competitivo de los Destinos Turísticos.

Del análisis de la tipología de las redes presentada en el epígrafe anterior y considerando que el sector turístico se conforma por la suma de las distintas actividades industriales y 
comerciales, que generan bienes y servicios, para ser consumidos entera o parcialmente por visitantes; se infiere que, las redes que deben propiciarse en el marco de la actividad turística son las confederadas, ya que en ellas se combina la cooperación con la competencia y se suscriben acuerdos formales e informales que sirven para dirigir acciones conjuntas y lograr los objetivos de desarrollo competitivo que persiguen los destinos turísticos.

En este marco referencial, Morrison y col. (2004) mencionan algunos de estos objetivos, clasificándolos en cuatro categorías generales, según se detalla:

Aprendizaje e intercambio: las redes de cooperación hacen posible un mayor flujo de información e intercambio de experiencias, entre los actores turísticos y la comunidad, aspecto que resulta favorable para lograr cambios en los valores culturales y afianzar los procesos de sensibilización y apertura turística en los habitantes de la localidad.

Comunidad: con el cambio de valores en los residentes, se refunda el sentido de comunidad, produciéndose por consiguiente, una mayor valorización del patrimonio y de los atractivos turísticos locales. Cuando una comunidad valora el patrimonio local (sea este natural o cultural, tangible o intangible), se incrementan de manera importante el nivel de conservación y el grado de atractivo de este patrimonio, aumentando la calidad del producto turístico global del destino y haciendo que la experiencia del turista resulte más positiva. Asimismo, las redes facilitan la inserción de los pequeños emprendedores locales en la actividad turística, aspecto que favorece el desarrollo turístico del destino.

Actividad empresarial: los vínculos que establecen las empresas al interactuar en redes, producen algunos beneficios individuales y colectivos que se extienden a la economía y sociedad en general, entre los que pueden destacarse: una fuerte cohesión, que resulta favorable para el fortalecimiento y aumento de la calidad del producto turístico global; el incremento de la eficiencia individual y mayor acceso a fuentes públicas y privadas de financiamiento, lo que a su vez funciona como estímulo extra para la conformación y el mantenimiento de las redes en los destinos turísticos.

Estructura económica: las ventajas que ofrecen las redes de cooperación a la estructura económica de un destino, se encuentran estrechamente vinculadas a todos los aspectos anteriores; por ejemplo, una comunidad que mejora su nivel de intercambio y aprendizaje, protege su patrimonio, y cuenta con empresas eficientes, tiene grandes posibilidades de desarrollarse económicamente. Por otro lado, una estrecha relación entre los agentes turísticos y económicos de la localidad que cooperan en redes, puede llevar a un mayor aprovechamiento de los recursos económicos locales, dinamizando la economía y potenciando a su vez el turismo como motor de desarrollo. De la misma forma, la creación de redes de emprendedores locales, permite reducir las fugas de capitales resultantes de las transacciones realizadas con agentes económicos de otras localidades y, consecuentemente, aumentaría la inversión interna, generando efectos positivos en toda la economía. 


\section{Consideraciones Finales}

Las redes de cooperación organizacional constituyen un sistema dinámico en el que se articulan actores para alcanzar objetivos colectivos e individuales, a través de la colaboración y el intercambio. Cuando este intercambio se lleva a cabo en el marco de la actividad turística, da lugar a las redes turísticas de cooperación, cuyos principales actores son las empresas turísticas, los organismos gubernamentales, las comunidades organizadas y otras organizaciones no estatales públicas y privadas.

Las redes se representan a través de formas abstractas, conformadas por nodos e interconexiones; siendo los nodos los agentes o actores sociales, dada su naturaleza de realizar alguna acción determinada; mientras que, las interconexiones, representan las relaciones de intercambio o de cooperación que se establecen entre los actores, señalando cuáles son los diferentes caminos que puede tomar el flujo de información, bienes o servicios a través de la red.

Las relaciones que se establezcan entre los nodos, a partir de sus interconexiones, puede dar lugar a redes con forma de estrella, malla o mixta; cada tipología con características particulares asociadas. Asimismo, se identifican en las redes tres propiedades: la intensidad relacional, que proporciona información acerca de la cantidad de vínculos de cada actor; la centralidad, que permite identificar a los actores con mayor capacidad de acción dentro de la red; y la conectividad, relacionada con la direccionalidad y fuerza de los vínculos.

En líneas generales, la creación de redes, responde a la necesidad de lograr cuatro metas: economía de escala, reducción de costos operativos, promoción de la integración entre los sectores público y privado, y el desarrollo de ventajas competitivas. Asimismo, en el contexto de la actividad turística, los objetivos de las redes van dirigidos a la creación y desarrollo de infraestructura turística, el diseño de planes para la promoción del turismo, la participación de los turistas como agentes promotores de los destinos $\mathrm{y}$, en general, hacia el fortalecimiento del producto turístico territorial.

\section{Bibliografía}

Astley, G. \& Fombrun, C. (1983). Collective Strategy: social ecology of organizational environments. Academy of Management Review. Vol. 8, $\mathrm{N}^{\circ}$ 4. 576-587. Obtenido de: https://www.jstor.org/stable/258259?seq=1\#page_scan_tab_contents

Becerra, F. \& Álvarez, C. (2011). El talento humano y la innovación empresarial en el contexto de las redes empresariales: El clúster de prendas de vestir en Caldas, Colombia. Estudios $\begin{array}{lllll}\text { Gerenciales } & N^{\circ} & 27 . & \text { 209-232. Obtenido }\end{array}$ http://www.redalyc.org/pdf/212/21220043010.pdf

Bonomie, M. \& Meleán, R. (2007). Redes empresariales como estrategia de cooperación en el sector avícola del Estado Zulia. Compendium $\mathrm{N}^{\circ}$ 19. 5-30. Obtenido de: http://www.ucla.edu.ve/dac/Compendium/revista19/01_Bonomie_Melean.pdf 
Cubero, R. (2005). Perspectivas Constructivistas. La intersección entre el significado, la interacción y el discurso. Colección crítica y fundamentos, Vol. 8. Barcelona, España: GRAO.

Fernández, J. \& Arranz, N. (2010). Las Redes de Cooperación Empresarial: ¿Una Organización para el Próximo Milenio?. Revistadyo.com. $\mathrm{N}^{\circ}$ 21. 12-19 Obtenido de: https://dialnet.unirioja.es/servlet/articulo?codigo $=169890$

Galán, J., Casanueva, C. \& Castro, I. (2010). Las relaciones empresariales: una tipología de redes. Innovar Journal Revista de Ciencias Administrativas y Sociales. Vol. 20, № 38. 27-44. Obtenido de: http://revistas.unal.edu.co/index.php/innovar/article/view/22286

Garrido, F. (1998). El análisis de redes en el desarrollo local. Madrid, España. Obtenido de: www.formacionciescoop.cl/wp-content/uploads/2011/08/Garrido-Análisis-de-redes.pdf

González, T. (2007). Redes de Cooperación Empresarial Internacionales Vs. Redes Locales. Revista Venezolana de Gerencia, Vol. 12, Nº. 37. 9 - 26.

Grandori, A \& Soda, G. (1995). Inter-firms Networks: antecedents, mechanisms and forms. Organ studs. Vol. $\quad 16, \quad \mathrm{~N}^{\mathrm{o}} 2 . \quad 183-214 . \quad$ Obtenido de: http://journals.sagepub.com/doi/pdf/10.1177/017084069501600201

Granovetter, M. (1973). The strength of weak ties. American Journal of Sociology. $N^{\circ} 78.1360$ $1380 . \quad$ Obtenido de: http://www.jstor.org/discover/10.2307/2776392?uid=3739296\&uid=2\&uid=4\&sid=2110 2741467813

Kornblit, A. (2007). Metodologías Cualitativas en Ciencias Sociales: Modelos y Procedimientos de Análisis. 2da. ed. Buenos Aires, Argentina: Editorial Biblos.

Morrison, A., Lynch, P. \& Johns, N. (2004). International tourism networks. International Journal of Contemporary Hospitality Management. Vol. 16, $\mathrm{N}^{\mathrm{o}}$ 3. 197-202. Obtenido de: http://www.emeraldinsight.com/journals.htm?articleid=867535\&show=abstract

Perego, L. (2003). Competitividad y Clústers Productivos. Madrid, España: Editorial Académica Española.

Sáez, D. \& Cabanelas, J. (1997). Cooperar para Competir con Éxito. Madrid, España: Ediciones Pirámide.

Trigueros, I., Mondragón, J. \& Serrano, M. (2001). Trabajador Social. Temario para la preparación de oposiciones. Vol. 1. Sevilla, España: Editorial MAD.

Valdéz, R. (2009). La formación de redes para la promoción de destinos turísticos: un análisis conceptual. Cuaderno virtual de turismo. Vol. 9, $\mathrm{N}^{\mathrm{o}} 3$. Obtenido de: http://www.redalyc.org/html/1154/115412543005/ 
Vázquez, A. (2005). Las nuevas fuerzas económicas del desarrollo. Barcelona, España: Antoni Bosch Editor. 